\title{
Use of soluble antigens in leprosy epidemiology
}

\author{
M D GUPTE \& D S ANANTHARAMAN \\ CJIL Field Unit for Epidemiology of Leprosy, 51, J.B. Estate, \\ Avadi, Madras 600 054, India
}

Accepted for publication 16 May 1988

\begin{abstract}
Summary Rees and Convit antigens prepared from armadillo-derived Mycobacterium leprae are presently available. This study was undertaken to understand the skin test reactions produced by these antigens in comparison to tuberculin. A standard tester skin-tested about 250 individuals. The indurations were read at the end of $48 \mathrm{~h}$ for Rees and Convit and at the end of $72 \mathrm{~h}$ for tuberculin by a standard reader who read these reactions following blind procedures again after $2 \mathrm{~h}$. The values of standard deviations for the mean differences were $1 \cdot 0,2 \cdot 6$ and $2.4 \mathrm{~mm}$ for tuberculin, Rees and Convit antigens respectively. Standard deviations for the mean differences for two different tests using the same antigen on the same individual twice, were $3 \cdot 0,6 \cdot 0$ and $5.3 \mathrm{~mm}$ respectively. Two batches of Rees antigen gave reasonably consistent results, but the skin-test readings with 2 batches of Convit antigen differed substantially. The available antigens need further improvement.
\end{abstract}

\section{Introduction}

The need for an antigen like tuberculin has been felt for a long time for the study of epidemiology of leprosy. ${ }^{1}$ Lepromin is a minimal vaccine and it cannot serve this purpose. ${ }^{2}$ Drs Convit and Rees have prepared soluble antigens from $M$. leprae of armadillo origin. ${ }^{3}$ These antigens are known af ter their names. Two batches of Convit and Rees antigens were supplied to our Unit by the IMMLEP programme of the World Health Organization. Several studies have been undertaken using these antigens. The present paper deals with the nature of the skin reactions produced by these antigens, reproducibility of the skin-test readings, and comparison of two different batches of these antigens with respect to skin-test reactions. It was necessary to undertake these studies as there is no reported research work on these aspects of skin-test reactions in leprosy. For the sake of comparison, tuberculin skin testing was also undertaken.

\section{Material and methods}

\section{STUDY POPULATION}

The skin-test antigen studies were conducted in 2 villages from Sriperumbudhur Taluk, Chingleput District, Tamil Nadu. Prevalence of leprosy in these 2 villages was over 50 per 1000 population examined. 


\section{ANTIGENS}

In most of the studies mentioned below, we used Rees antigen (batch CD19) and Convit antigen (batch SA-IND, 1-16-86). For studying batch variations, batch Wel-3-CD73 of Rees antigen and batch IB-Lote-2 4-6-87 of Convit antigen were also used. These antigens were received from Drs Rees and Convit, through the courtesy of IMMLEP, TDR, WHO. Tuberculin PPD (RT 23, 1 TU per dose) was used for comparisons and was procured from the BCG Laboratory, Madras, India.

Rees antigen is a soluble antigen preparation of gamma irradiated $M$. leprae, purified according to IMMLEP protocol, with particulate material removed. ${ }^{3}$ The Rees antigen preparations contained $10 \mu \mathrm{gms}$ protein/ml for both the batches used.

Convit antigen is also a soluble antigen and is prepared from 'live' $M$. leprae. Suspensions of $M$. leprae are passed through a standard French press. The extract is centrifuged and filtered following standard procedures. Preparations after necessary dilutions are autoclaved. ${ }^{3}$

\section{SKIN TESTING AND READING}

The skin-test antigens were given by superficial intracutaneous injections on the dorsum and the volar side of the forearm. The specified quantity, $0 \cdot 1 \mathrm{ml}$, was measured according to the markings on the barrel of a tuberculin syringe. We used Omega tuberculin syringes, and $26 \mathrm{G}, 3 / 8^{\prime \prime}$ platinum needles, supplied by IMMLEP, TDR, WHO. Each skin test was read at specified intervals, the reaction was felt, and if the induration was present its transverse diameter was measured. All measurements were made with a ruler calibrated in millimetres. Each palpable reaction was also examined for the density of induration. We adopted these standard procedures as they are followed for the tuberculin testing and reading. ${ }^{4}$

The following studies were undertaken to standardize various procedures in Rees and Convit skin tests. A standard tuberculin tester and a standard tuberculin reader carried out all the testings and readings reported below. For all of these studies the reader was unaware of the identity of the skin-test antigen while reading a reaction. This blinding was achieved by providing him with a secretary at the time of readings.

\section{Study 1 Optimum interval for skin-test readings}

Tuberculin skin tests were read at the end of $72 \mathrm{~h}$. Reactions following Rees and Convit antigen skin tests were measured at 24-, 48- and 72-h intervals in a set of 767 individuals. It was possible to make all 3 readings for both Rees and Convit antigens in 471 individuals. These findings are given in

Table 1. Skin-test readings for Rees and Convit antigens at 24-, 48- and 72-hr interval in 471 individuals.

\begin{tabular}{|c|c|c|}
\hline \multirow[b]{2}{*}{ Interval (h) } & \multicolumn{2}{|c|}{ Reactions } \\
\hline & Rees antigen & Convit antigen \\
\hline & \multicolumn{2}{|c|}{ Mean $\pm \mathrm{SD}(\mathrm{mm})$} \\
\hline 24 & $11 \cdot 47 \pm 5 \cdot 71$ & $9 \cdot 52 \pm 4 \cdot 98$ \\
\hline 48 & $11 \cdot 00 \pm 6 \cdot 17$ & $10 \cdot 04 \pm 5 \cdot 44$ \\
\hline \multirow[t]{2}{*}{72} & $9 \cdot 41 \pm 5 \cdot 84$ & $8 \cdot 61 \pm 5 \cdot 30$ \\
\hline & \multicolumn{2}{|c|}{ Paired 't' values } \\
\hline 24 vs 48 & 1.95 & $2 \cdot 73$ \\
\hline 48 vs 72 & $7 \cdot 54$ & $7 \cdot 49$ \\
\hline
\end{tabular}


Table 1. There was a marginal difference in $24-$ and 48 -h skin-test readings, $72-\mathrm{h}$ readings were significantly lower when compared with the 48 -h readings. The findings were similar when all the available skin-test readings were considered. It was decided to use the 48-h readings, in pref erence to $24 \mathrm{~h}$ readings, to avoid the early nonspecific response.

\section{Study 2 Intrareader variations}

Rees and Convit antigens $(0 \cdot 1 \mathrm{ml}$ of each) were injected in the dorsum of the forearms, allocation of the antigens to right or left forearms was done randomly. The reader read all the tests twice at an interval of approximately $2 \mathrm{~h}$. The initial part of the study (April 1987) involved approximately 250 individuals.

It was felt desirable to document the intrareader variations in reading tuberculin tests for the sake of comparison with Rees and Convit reactions. This part of the study was also done with approximately 250 individuals.

Following these initial exercises, along with the interbatch variation studies, the standard reader was asked to repeat the same exercise for Rees and Convit antigens in a limited number of individuals. Eighty-six and 61 paired observations for Rees and Convit skin-test readings were made. This work was done in July 1987, 3 months after the initial intrareader variation study.

Findings for the intrareader variations are given in Table 2.

\section{Study 3 Intertest variations}

Two skin tests of $0 \cdot 1 \mathrm{ml}$ of Rees and Convit antigens were perf ormed on the selected individuals on the same day. Readings were made after $48 \mathrm{~h}$. Approximately 200 individuals were involved in Rees and Convit antigen studies. Tuberculin exercise was essentially for comparison and was done in about 300 individuals. The selected individuals were retested with the same antigen previously used in them after a gap of approximately 1 month. Findings of this study are given in Table 3.

\section{Study 4 Interbatch variations}

This study was done in two parts. In the first part, 2 batches each of Rees and Convit antigens were skin tested in two different groups of individuals. This part of the study brought out distinct batch variations for Convit antigen. It was felt desirable to repeat this study in one group of individuals who received all the 4 skin tests for 2 batches each of Rees and Convit antigens. Injection sites for the 4 skin tests were selected on the basis of randomization. The findings were confirmed (Table 4 , Figure 1).

Table 2. Intrareader variations - tuberculin, Rees and Convit antigens.

\begin{tabular}{|c|c|c|c|c|c|}
\hline & \multirow{2}{*}{$\frac{\text { Tuberculin }}{\text { June } 87}$} & \multicolumn{2}{|c|}{ Rees } & \multicolumn{2}{|c|}{ Convit } \\
\hline & & April 87 & July 87 & April 87 & July 87 \\
\hline Number & 252 & 250 & 86 & 251 & 61 \\
\hline \multicolumn{6}{|l|}{ Mean reactions (mm) } \\
\hline I reading & $13 \cdot 27$ & $14 \cdot 83$ & $15 \cdot 17$ & $13 \cdot 37$ & $10 \cdot 26$ \\
\hline II reading & $13 \cdot 18$ & $15 \cdot 13$ & $14 \cdot 94$ & $13 \cdot 90$ & $10 \cdot 31$ \\
\hline Mean difference (đ) ( $\mathrm{mm})$ & $0 \cdot 09$ & $0 \cdot 30$ & $0 \cdot 23$ & 0.53 & 0.05 \\
\hline Standard deviation $(\mathrm{d})(\mathrm{mm})$ & $1 \cdot 00$ & $2 \cdot 60$ & $1 \cdot 21$ & $2 \cdot 40$ & $1 \cdot 30$ \\
\hline
\end{tabular}


Table 3. Intertest variations.

\begin{tabular}{lcrr}
\hline & Tuberculin & Rees & Convit \\
\hline Number & 314 & 191 & 204 \\
Mean reactions (mm) & & & \\
$\quad$ Test I & 12.24 & 10.93 & 9.82 \\
$\quad$ Test II & 13.00 & 11.08 & 10.80 \\
Meandifference (d) $(\mathrm{mm})(\mathrm{II}-\mathrm{I})$ & +0.76 & +0.15 & +0.98 \\
Standard deviation $(\mathrm{d})(\mathrm{mm})$ & 2.98 & 5.96 & 5.32 \\
Probability & $<0.05$ & $>0.05$ & $<0.05$ \\
& & & \\
\hline
\end{tabular}

Table 4. Interbatch variations for two* batches of Rees and Convit antigens (1987).

\begin{tabular}{|c|c|c|c|c|c|c|c|}
\hline \multirow[b]{2}{*}{ Antigens } & & \multirow{2}{*}{$\begin{array}{l}\text { No. } \\
\text { tested }\end{array}$} & \multicolumn{2}{|c|}{ Mean reactions } & \multirow{2}{*}{$\begin{array}{l}\mathrm{d}(\mathrm{mm}) \\
{[\mathrm{I}-\mathrm{II}]}\end{array}$} & \multirow{2}{*}{$\begin{array}{l}\text { SD } \\
\text { for } \bar{d}\end{array}$} & \multirow{2}{*}{$\begin{array}{c}\text { Correlation coefficient } \\
\text { for I and II }\end{array}$} \\
\hline & & & Batch I & Batch II & & & \\
\hline \multirow[t]{2}{*}{ Convit } & Expt 1 & 74 & $10 \cdot 64$ & $7 \cdot 88$ & $2 \cdot 76$ & $5 \cdot 47$ & $0 \cdot 53$ \\
\hline & Expt 2 & 95 & $12 \cdot 33$ & $9 \cdot 38$ & 2.95 & $4 \cdot 73$ & $0 \cdot 66$ \\
\hline \multirow[t]{2}{*}{ Rees } & Expt 1 & 94 & $15 \cdot 50$ & 14.97 & 0.53 & $2 \cdot 82$ & $0 \cdot 81$ \\
\hline & Expt 2 & 95 & $14 \cdot 94$ & $14 \cdot 63$ & $0 \cdot 31$ & $3 \cdot 65$ & $0 \cdot 77$ \\
\hline
\end{tabular}

* Rees antigen batches: I. CD19; II. Wel-3 CD73. Convit antigen batches: I. SA-IND, 1-16-86; II. IB-Lote-4-6-87.

\section{Observations and discussion}

\section{INTRAREADER VARIATIONS}

It could be seen from Table I that the readings for the tuberculin indurations were very close to each other, the mean difference being $0.09 \mathrm{~mm}$, with a standard deviation of $1.00 \mathrm{~mm}$. The difference in the readings of the same reaction twice was within $\pm 2 \mathrm{~mm}$ on $95 \%$ of the observations.

With respect to Rees and Convit antigens, the initial intrareader variation was fairly wide with standard deviations of 2.60 and $2.40 \mathrm{~mm}$ respectively. It is worth noting that this amount of variation was seen in a standard tuberculin reader. As could be seen from the small number of paired observations, 3 months later, the standard deviations came down to 1.21 and $1.30 \mathrm{~mm}$ for Rees and Convit antigen readings.

\section{INTERTEST VARIATIONS}

Table 3 brings out the marked diff erences between tuberculin and the leprosy soluble antigens. With tuberculin, the value of standard deviation for mean difference between two tests was $2.98 \mathrm{~mm}$. Ninety-five per cent of the observations were within $\pm 6 \mathrm{~mm}$ of the mean difference. These observations are similar to those quoted by Rajnarain in standard tuberculin readers. ${ }^{5}$ With respect to the soluble antigens, the standard deviations were 5.96 and $5.32 \mathrm{~mm}$. It is expected that this difference also would come down with experience. 


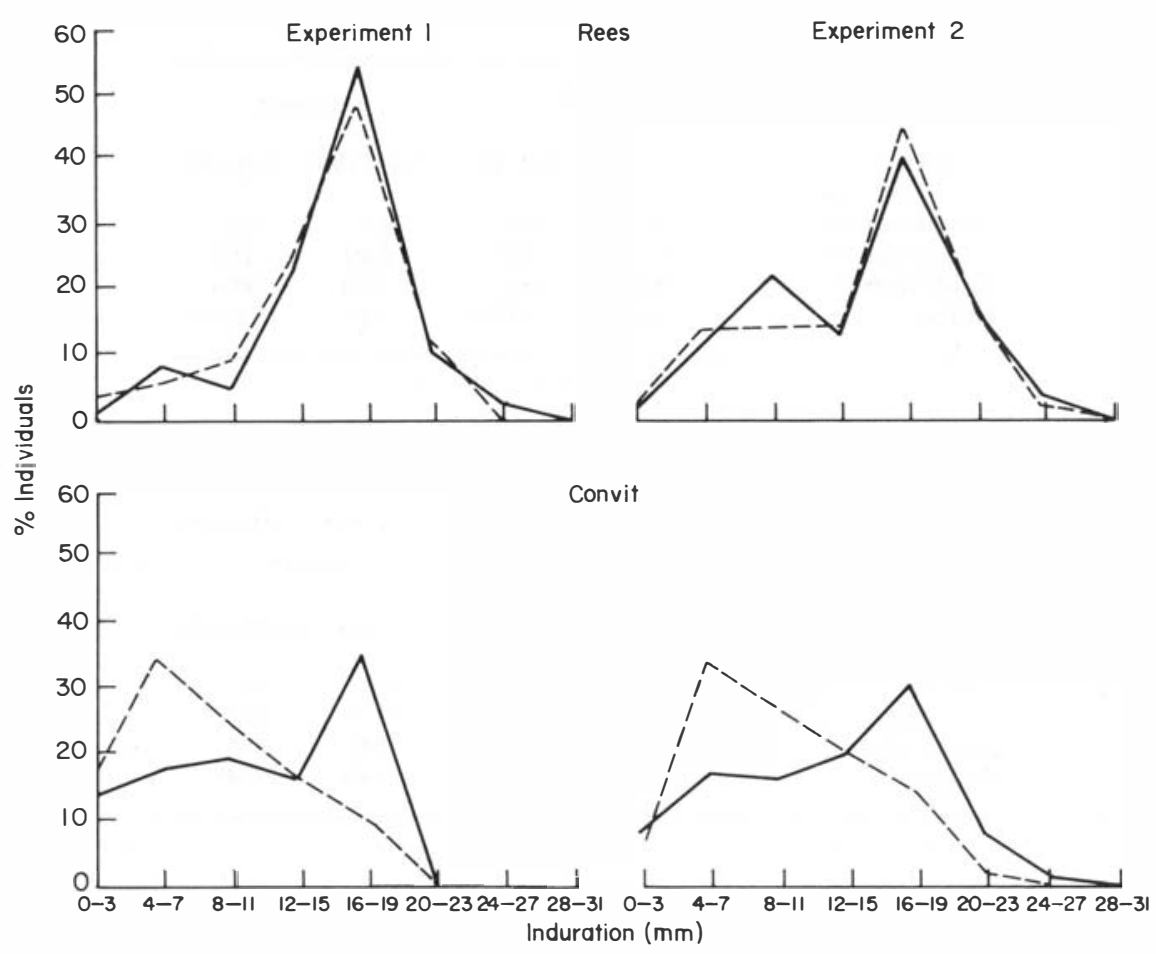

Figure 1. Interbatch variations: Rees and Convit skin-test reactions. - , Batch I*; ----- , Batch II. * Rees-Batch I: CD19; Batch II: WEL-3-CD73. Convit-Batch I: SA-IND 1-16-86; Batch II: IB-Lote 4-6-87.

\section{INTERBATCH VARIATION}

Two batches each of Rees and Convit antigens were tested in two different experiments. Information from Table 4 and the frequency distributions from Figure 1, clearly bring out the differences between 2 batches of Convit antigen. Both these batches were prepared following similar procedures and were standardized in Venezuela by Dr Convit (personal communication). The reason for the difference observed by us is difficult to understand.

\section{SKIN TEST REACTIONS TO THE SOLUBLE LEPROSY ANTIGENS}

In the process of the various studies mentioned above, the density of the indurations to the soluble antigens were also recorded. The scale adopted for this was arbitrary. Soft reactions meant reactions merging almost imperceptibly with the normal skin around. Hard reactions were quite distinct in the margins. When we looked for the density of indurations due to Rees and Convit antigens in the initial stages of the present work (1239 indurations), about $30 \%$ of them were 'soft', $30 \%$ hard and the remaining $40 \%$ were in between and did not pose difficulty in reading them to the standard tuberculin reader. Reactions to tuberculin were almost invariably hard or firm in consistency. The reason for this soft consistency is not known. This soft consistency of the 
Table 5. Implications on positivity due to intrareader variations.

\begin{tabular}{lccccc}
\hline & \multicolumn{2}{c}{ Rees } & & \multicolumn{2}{c}{ Convit } \\
\cline { 2 - 3 } \cline { 5 - 6 } Antigens & April 1987 & July 1987 & & April 1987 & July 1987 \\
\hline Number of individuals & 250 & 86 & & 251 & 61 \\
\% positives by both & $69 \cdot 2$ & $83 \cdot 7$ & & $64 \cdot 1$ & $45 \cdot 9$ \\
Concordance & $90 \cdot 4$ & $96 \cdot 5$ & & $92 \cdot 0$ & $98 \cdot 4$ \\
Kappa & $0 \cdot 75$ & $0 \cdot 86$ & & $0 \cdot 82$ & 0.97 \\
\hline
\end{tabular}

Table 6. Implications on positivity due to intertest and interbatch variations.

\begin{tabular}{lccccc}
\hline & \multicolumn{2}{c}{ Rees } & & \multicolumn{2}{c}{ Convit } \\
\cline { 2 - 3 } \cline { 5 - 6 } Antigens & Intertest & Interbatch & & Intertest & Interbatch \\
\hline Number of individuals & 191 & 95 & & 204 & 95 \\
\% positives by both & $30 \cdot 9$ & $63 \cdot 2$ & & $31 \cdot 4$ & $30 \cdot 5$ \\
Concordance & $73 \cdot 3$ & $85 \cdot 3$ & & $75 \cdot 0$ & $69 \cdot 5$ \\
Kappa & 0.46 & $0 \cdot 65$ & & 0.49 & 0.43 \\
\hline
\end{tabular}

indurations, makes them extremely difficult for the correct and consistent reading even for an experienced skin-test reader.

The experience of Ponnighaus and Fine in Malawi using various batches of Rees antigen and a smaller number of Convit-type antigens has been reported ${ }^{3}$ (TDR, IMMLEP). They found changes in the skin reactivity to these antigens in a time span of 3 months. Part of these 'changes' could be on account of the inherent nature of these antigens, which produce comparatively sof ter reactions.

\section{IMPLICATIONS OF VARIATIONS IN REES AND CONVIT SKIN TEST READINGS}

Observed variations in the skin test readings are expected to lead to variable interpretation of the skin tests in terms of positives and negatives. In the initial exercise for the intrareader variations, the magnitude of this kind of error was not very high. It was further possible to reduce this difference substantially with experience (Table 5). Efforts to read positive and negative reactions in the intertest and interbatch experiments were almost frustrating (Table 6).

\section{Conclusions}

Comparatively recent availability of soluble antigens in leprosy have raised the possibility of promising research in leprosy epidemiology. However very little published work is available documenting the methods needed for skin testing and reading these antigens. The studies reported here bring out certain problems in reading these reactions consistently. This difficulty could be partly on account of the softness of indurations produced by these antigens. It is possible to reduce the intrareader variation with experience. However, there is a continued requirement for research on standardizing and improving the presently available soluble skin test antigens in leprosy. It is also necessary to produce better skin test antigens than the ones presently available. 


\section{Acknowledgments}

The authors are gratef ul to Drs Rees and Convit for the supply of Rees and Convit antigens and also to IMMLEP, TDR, World Health Organization, for arranging the supply of antigens to us. The authors appreciate the statistical assistance by $\mathrm{Mr} \mathrm{S}$ Kannan and the secretarial assistance by $\mathrm{Mr}$ N K S Brahaspathy.

\section{References}

1 Ten Dam HG, Sansarricq H. The use of immunological tests in epidemiological work. Symposium on epidemiology of leprosy, Geilo, Norway, 1981. Lep. Rev, 1981; 52: (Suppl. 1), 289-98.

${ }^{2}$ Testing of purified armadillo-derived $M$. leprae in man (Document finalized by the IMMLEP Steering Committee at its meeting, 10-12 June 1981). TDR/IMMLEP/SC/Test/81.1: p. 2.

3 Vaccination trials against leprosy: A meeting of the epidemiology Subgroup of Scientific Working Group on the immunology of leprosy, Geneva, 11-13 Feb. 1985; TDR/IMMLEP/EPD/85.3: p. 7-8.

${ }^{4}$ Edward LB, Palmer CE, Magnus K. BCG Vaccination, World Health Organization, 1953; Monograph Series No. 12: p. 32-3.

${ }^{5}$ Raj Narain, Nair SS, Chandrasekhar P, Ramanatha Rao G. Problems connected with estimating the incidence of tuberculosis infection. Bull WHO, 1966; 34: 605-22. 